\title{
Prevalance of Streptococcus pyogenes infection in children aged between 5 to 15 years with acute tonsillopharyngitis and its antibiogram.
}

\author{
Sanjeeb Sharma ${ }^{1}$, Sh. Praveen ${ }^{2}$, Kh. Sulochana Devi ${ }^{3}$, Biswajeet Sahoo ${ }^{1}$, \\ Waikhom Shashi Singh ${ }^{1}$, Thokchom Devaraj Singh ${ }^{1}$ \\ ${ }^{l}$ Post graduate student, Department of Microbiology, Regional Institute of Medical Sciences, Imphal, Manipur, \\ India. \\ ${ }^{2}$ Associate Professor, Department of Microbiology. \\ ${ }^{3}$ Professor, Department of Microbiology.
}

\begin{abstract}
:
Objective: To study the prevalence of group A beta haemolytic streptococcal (GABHS) infection among children aged between 5 to 15 years suffering from acute tonsillopharyngitis and its antibiogram.

Research Design And Methods: This was a crossectional prospective study done over two years, by procuring data from the total 123 patient records of OPD patients. The clinical settings including history, examination and the throat swab cultures with anti-microbial sensitivity testing performed under standard conditions.

Results: In this study, Streptococcus pyogenes was isolated in 21 patients out of the total 123 patients studied (17.1\%), out of which 14(11.4\%) were isolated from the age group between 11-15 years and only 7(5.7\%) in the age group between 5-10 years. Males (10.6\%) predominated in comparision to females $(6.5 \%)$ with male female ratio of 1.6:1 and all the isolates were 100\% sensitive to cefaclor, penicillin-G and ampicillin followed by $38.1 \%$ resistance to erythromycin, $28.6 \%$ to azithromycin and $33.3 \%$ to tetracycline respectively.

Conclusion: The current study showed $17.1 \%$ prevalence of GABHS in the study population suffering from acute tonsillopharyngitis and also showed significant amount of resistance against commonly used antibiotics which were earlier sensitive probably due to injudicious use of antibiotics without undergoing routine culture and sensitivity.
\end{abstract}

Keywords: acute tonsillopharyngitis, GABHS (Group A beta haemolytic Streptococci), resistance

\section{Introduction:}

Group A beta-hemolytic streptococcus (GABHS) is the most important of the bacterial causes of acute pharyngitis accounting for $15 \%$ to $30 \%$ of cases in children ${ }^{[1]}$. Untreated patients may develop purulent complications including otitis media, sinusitis, peritonsillar and retropharyngeal abscesses and cervical adenitis. The significance of respiratory tract infection is related to particular to its acute morbidity and its non suppurative sequelae that is acute rheumatic fever and acute glomerulonephritis. Acute rheumatic fever continues to be a health problem. Reappearance of acute rheumatic fever in several areas serves as a reminder of the importance of its continued attention to its prevention in the developing as well as developed countries. Transmission rates of group A streptococcal infection is approximately $35 \%$ within a family or school if a patient is untreated and the carrier rate is different in developed and developing countries ${ }^{[2]}$. It is usually spread by direct person to person contact mostly likely via drops of saliva or nasal secretions. Respiratory droplets are the usual mechanism of spread because this organism primarily localises in the throat ${ }^{[3]}$.

Streptococcus pyogenes strains still continue to be penicillin susceptible, but erythromycin resistance has increasingly been reported. A recent study ${ }^{[4]}$ has documented that $2.1 \%$ of S. pyogenes strains collected were macrolide resistant. Significant rates of erythromycin resistance have been reported in many countries including Finland, Sweden, Spain, France, and Italy ${ }^{[5,6]}$. If properly timely intervention is undertaken by the physician for diagnosis of streptococcal pharyngitis by normal conventional methods then adequate timely treatment can be possible with the proper use of antibiotics and prevent the long term complications like acute rheumatic fever and Rheumatic heart disease which has a prevalence of 4 to 6 per 1000 children per year in the developing countries ${ }^{[7]}$.

\section{Objective:}

To study the prevalence of group A beta haemolytic streptococcal (GABHS) infection among children aged between 5 to 15 years suffering from acute tonsillopharyngitis and its antibiogram by standard modified Kirby Bauer disc diffusion method to the commonly used antibiotics. 


\section{Source of data:}

\section{Material And Methods:}

Data were collected from the preformed performa prepared from OPD patient suffering from acute tonsillopharyngitis aged between 5 to 15 years over the past two years, in a tertiary health care centre in Northeastern India.

A prospective cross sectional descriptive study was done over two years by collecting records of patients between the 1st of November 2012 to October 2014 at Regional Institute of Medical Sciences, Imphal Manipur India. A minimum of 123 patients diagnosed as acute tonsillopharyngitis by history and clinical examination was included in the study.

Detailed history and examination findings, laboratory investigations, the species of microorganisms and sensitivity to antibiotics used in the hospital were documented on a preformatted data sheet.

A sample size of 123 was selected using purposive sampling technique based on inclusion and exclusion criteria.

Inclusion criteria:

- Children between age 5 to 15 years who are willing and whose parents have permitted them to take part in the study.

- Children presenting with symptoms of acute throat infection irrespective of sex, religion, caste and socioeconomic status like fever greater than or equal to $38.5^{\circ} \mathrm{C}$ difficulty in swallowing, hoarseness, cough, malaise, generalized bodyache, loss of appetite and enlarged lymph nodes.

Exclusion criteria:

- Study population already on antibiotic treatment.

- Data analysis: Collected data were analyzed by ratios and percentages.

Absolute confidentiality of the individuals was maintained in this study.

Ethical approval was obtained from the institutional ethics Committee, RIMS Imphal, Manipur.

\section{Method of collection of data:}

Sample and sampling technique: Two swabs ${ }^{[8]}$ were taken from the throat of the study sample by sterile throat swabs. Routinely, the swab was rubbed over each tonsillar area and the posterior pharyngeal wall showing any exudates. Each specimen (swab) was processed by:

- Direct smear of throat swab was made and gram staining was done with its appropriate interpretation ${ }^{[9]}$.

- Immediately inoculating the specimen in 5\% sheep blood agar plate and then incubated under 5-10\% carbon dioxide (candle jar) at $37^{\circ} \mathrm{c}$ for 24 hours or up to 48 hours.

- Identification and confirmation of group A beta haemolytic streptococci was done by seeing the beta (complete) hemolysis pattern on the sheep blood agar plate, gram stain morphology showing gram positive cocci in chains, typical fine translucent colony morphology, non motile on motility testing and biochemical tests like negative catalase test, positive PYR hydrolysis tests and Bacitracin sensitivity test ${ }^{[10]}$.

All the identified Group A streptococcal isolates from throat swabs were subjected to invitro susceptibility test by modified Kirby-Bauer disc diffusion method on Mueller Hinton agar (MHA) containing 5\% defibrinated sheep blood as per CLSI (Clinical and Laboratory Standards Institute) guidelines. Pattern of susceptibility of the organism to the different antibiotics were noted down as per standard norm ${ }^{[11]}$. The antibiotics used in the study were tetracycline $(30 \mu \mathrm{g})$, erythromycin $(15 \mu \mathrm{g})$, cefaclor $(30 \mu \mathrm{g}), \quad$ penicillin-G (10units), ampicillin $(10 \mu \mathrm{g}) \quad$ and azithromycin $(15 \mu \mathrm{g})$ respectively ${ }^{[12,13]}$.

\section{Results:}

In this study out of 123 patients with acute tonsillopharyngitis who participated in the study, Streptococcus pyogenes was isolated in 21 patients out of total 123 patients (17.1\%) studied. (Fig. A,B,C)

Table 1: Age distribution of positive patients studied

\begin{tabular}{|c|c|c|c|}
\hline $\begin{array}{c}\text { Age in } \\
\text { years }\end{array}$ & $\begin{array}{c}\text { No. of patients } \\
\text { with positive } \\
\text { GABHS }\end{array}$ & $\begin{array}{c}\text { \% with } \\
\text { positive } \\
\text { GABHS }\end{array}$ & $\begin{array}{c}\text { \% } \\
\text { Total }\end{array}$ \\
\hline $5-10$ & 7 & $5.7 \%$ & $35.0 \%$ \\
\hline $11-15$ & 14 & $11.4 \%$ & $65.0 \%$ \\
\hline Total & 21 & $17.1 \%$ & $100 \%$ \\
\hline
\end{tabular}


Table 1 shows age wise distribution of the patients studied who were positive for GABHS with 7 patients $(5.7 \%)$ in the age group between 5-10 years and 14 patients $(11.4 \%)$ out of the total in the age group between 11-15 years respectively. Also the gender distribution ratio was 1.6:1 with 13 male patients $(10.6 \%)$ and 8 female patients $(6.5 \%)$ and Hindus were among the majority with total 12 isolates $(9.7 \%)$ followed by 5 $(4.1 \%)$ in Muslims and 4 (3.3\%) in Christians respectively based on religion. According to Kuppuswamy socioeconomic status scale $2012^{[14]}, 9$ patients $(7.3 \%)$ out of the total population (123) were from lower class, 6 $(4.9 \%)$ from upper middle class, $5(4.1 \%)$ were from middle class respectively. No positive cases were seen from the upper socioeconomic class group.

Table 2 A- Sensitivity and Resistance pattern of drugs studied for the confirmed isolates of GABHS in

\begin{tabular}{|l|c|c|c|c|}
\hline \multirow{3}{*}{ Antibiotics } & \multicolumn{3}{|c|}{ age between 5 to 10 years } \\
\cline { 2 - 5 } & \multicolumn{2}{|c|}{$\begin{array}{c}\mathbf{5 - 1 0} \\
(\mathbf{n}=7)\end{array}$} & \multicolumn{2}{c|}{$\begin{array}{c}\text { Total } \\
(\mathbf{n}=\mathbf{2 1})\end{array}$} \\
\cline { 2 - 5 } & Resistance & Sensitive & Resistance & Sensitive \\
\hline A.Penicillin & 0 & $7(100.0 \%)$ & $0(0 \%)$ & $21(100 \%)$ \\
\hline B.Azithromycin & $2(28.6 \%)$ & $5(71.4 \%)$ & $6(28.6 \%)$ & $15(71.4 \%)$ \\
\hline C.Erythromycin & $4(57.1 \%)$ & $3(42.9 \%)$ & $8(38.1 \%)$ & $13(61.9 \%)$ \\
\hline D.Cefaclor & 0 & $7(100.0 \%)$ & $0(0 \%)$ & $21(100.0 \%)$ \\
\hline E.Tetracycline & $6(85.7 \%)$ & $1(14.3 \%)$ & $14(66.7 \%)$ & $7(33.3 \%)$ \\
\hline F.Ampicillin & 0 & $7(100.0 \%)$ & $0(0 \%)$ & $21(100.0 \%)$ \\
\hline
\end{tabular}

Fig. 2A- Bar diagram showing Sensitivity and Resistance pattern of drugs studied for the confirmed isolates of GABHS for the age between $5-10$ years in the study population.

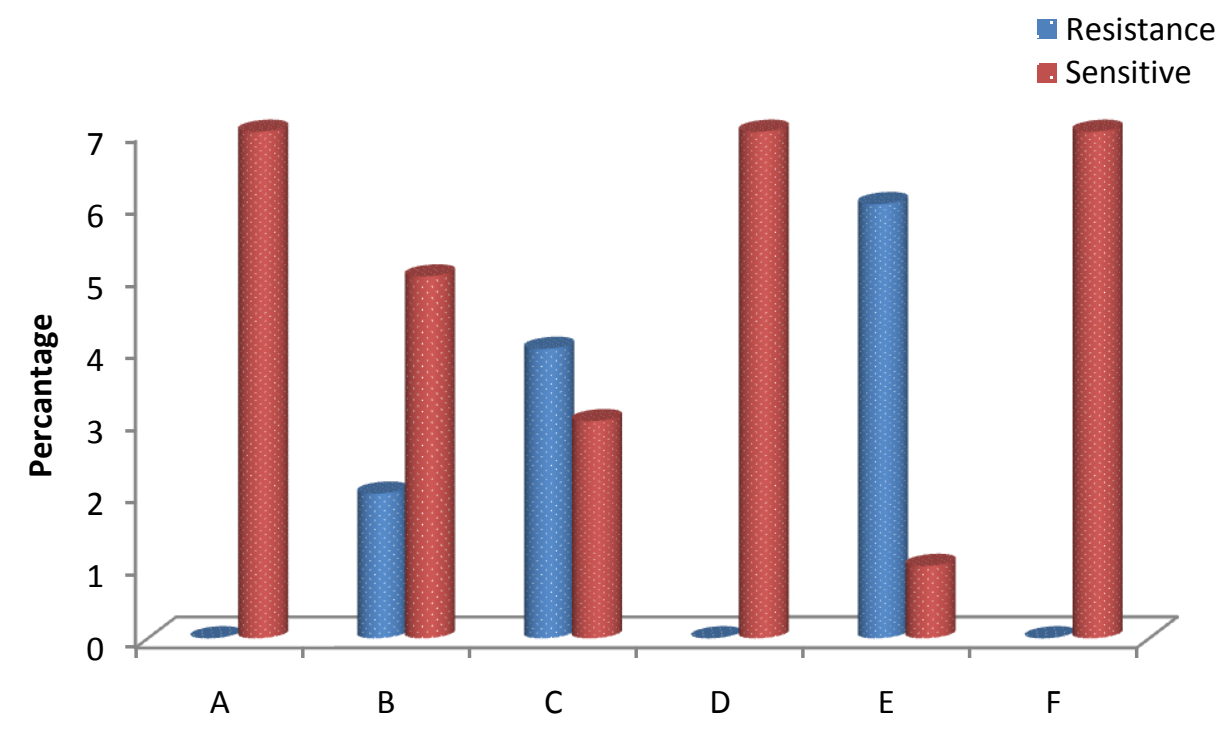

Table and fig. 3- Bar diagram showing Sensitivity and Resistance pattern of drugs studied for the confirmed isolates of GABHS for the age between $11-15$ years in the study population.

\begin{tabular}{|c|c|c|c|c|c|}
\hline & \multirow[t]{2}{*}{ Antibiotics } & \multicolumn{2}{|c|}{$\begin{array}{c}11-15 \\
(n=14)\end{array}$} & \multicolumn{2}{|c|}{$\begin{array}{c}\text { Total } \\
(\mathrm{n}=21)\end{array}$} \\
\hline & & Resistance & Sensitive & Resistance & Sensitive \\
\hline A. & Penicillin & 0 & $14(100.0 \%)$ & $0(0 \%)$ & $21(100 \%)$ \\
\hline B. & Azithromycin & $4(28.6 \%)$ & $10(71.4 \%)$ & $6(28.6 \%)$ & $15(71.4 \%)$ \\
\hline C. & Erythromycin & $4(28.6 \%)$ & $10(71.4 \%)$ & $8(38.1 \%)$ & $13(61.9 \%)$ \\
\hline D. & Cefaclor & 0 & $14(100.0 \%)$ & $0(0 \%)$ & $21(100.0 \%)$ \\
\hline E. & Tetracycline & $8(57.1 \%)$ & $6(42.9 \%)$ & $14(66.7 \%)$ & $7(33.3 \%)$ \\
\hline F. & Ampicillin & 0 & $14(100.0 \%)$ & $0(0 \%)$ & $21(100.0 \%)$ \\
\hline
\end{tabular}




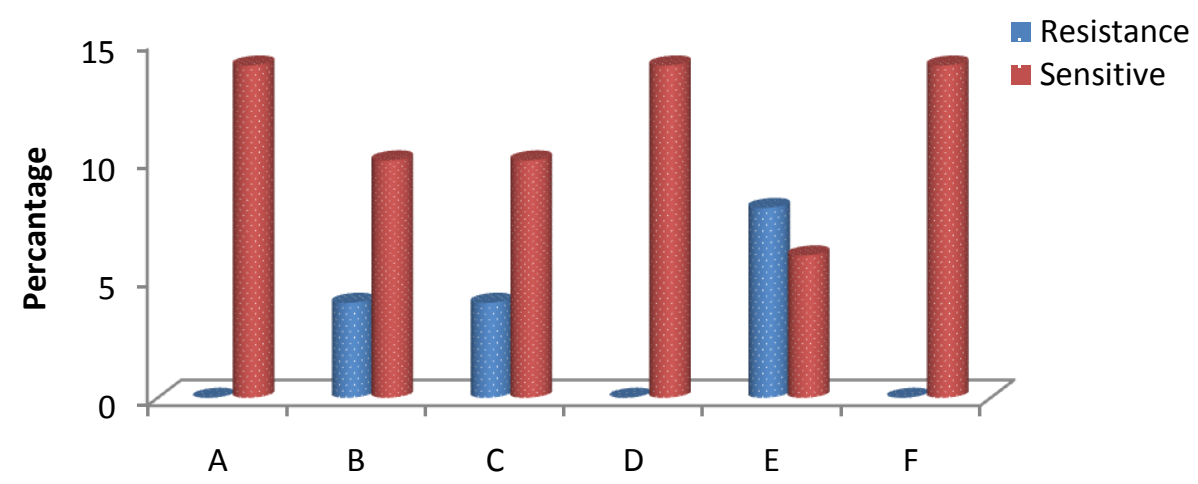

All the isolates were $100 \%$ sensitive to cefaclor, penicillin-G and ampicillin and $38.1 \%$ resistance pattern were seen to erythromycin followed by $28.6 \%$ to azithromycin and $33.3 \%$ to tetracycline respectively. (Fig.D)

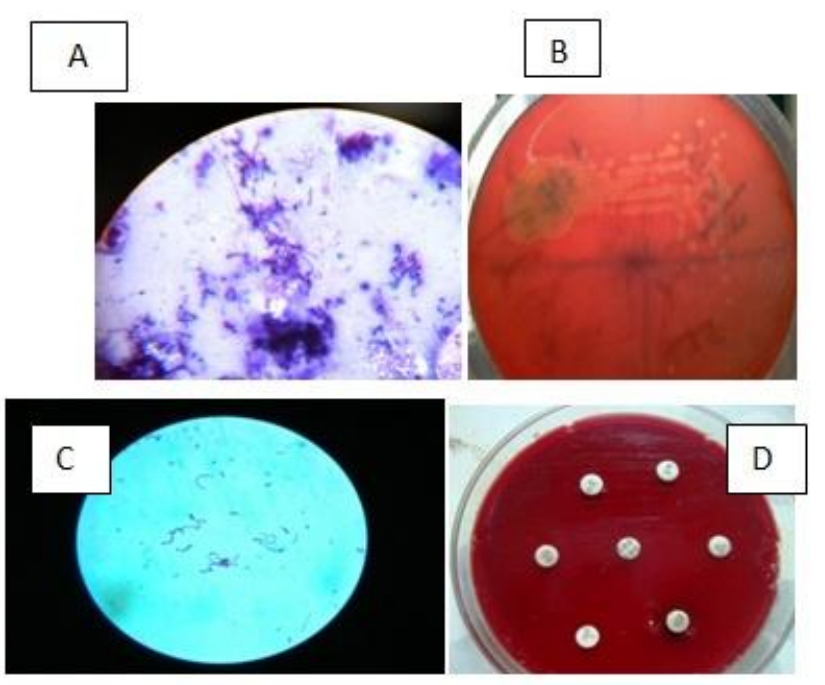

Fig.A-Streptococcus pyogenes (Gram positive cocci in chains) by Gram stain - Direct smear (1000x)

Fig.B- Colonies of Streptococcus pyogenes on sheep blood agar

Fig.C- Streptococcus pyogenes (Gram positive cocci in chains) by Gram stain - (1000x)

Fig.D- In-vitro antibiotic susceptibility test by modified Kirby-Bauer disc diffusion method on Mueller Hinton agar (MHA) containing 5\% sheep blood for isolated Streptococcus pyogenes

In the present study, Streptococcus pyogenes was isolated in 21/123 (17.1\%) patients, out of which 14 $(17.5 \%)$ were from the age group between 11-15 years and 7(16.3\%) from the age group between 5-10 years. This is in accordance with the previous studies conducted by Basili A et al ${ }^{[15]}$ which showed overall prevalence of GABHS as 17\% and the highest isolation rate was reported in children aged 11-15 years as in case of our study. Giir E et al ${ }^{[16]}$ also showed similar prevalence of GABHS (16.5\%) in his study with more male predominance $(52.2 \%)$ as well as study conducted by Gupta $\mathrm{R}$ et al ${ }^{[17]}$ which showed prevalence of $13.7 \%$ respectively. Prevalance was more in the age group of 11-15 years (11.4\%) than 5-10 years (5.7\%) which is similar with the findings of a study by Bassili $\mathrm{A}$ et al ${ }^{[15]}$. Contrast to this study, higher prevalence of Streptococcus pyogenes were seen in the age group between 5-10 years in studies conducted by Lin MH et al ${ }^{[18]}$ and Gupta $\mathrm{R}$ et $\mathrm{al}^{117]}$. Davies HD et al ${ }^{[19]}$ and Gunnarssu RK et al ${ }^{[20]}$ found that children were more prone to invasive Group A streptococcal infection than the general population due to immaturity of the immune system.

Prevalance of group A streptococcal infection were more in males (10.6\%) in comparison to females $(6.5 \%)$ out of the total study population, which has similar findings with the study conducted by Moses EA et al 
${ }^{[21]}$ and Giir E et al ${ }^{[16]}$. Contrast to this study, females outnumbered males in studies conducted by Gupta R et al [17]. Although numerous studies have no male- female disparity in the prevalence rate of group A streptococcal infection like study conducted by Madha $\mathrm{S}^{[22]}$ and Rijal K R et al ${ }^{[23]}$ respectively.

It was found that all the isolates of Streptococcus pyogenes were $100 \%(21 / 21)$ sensitive to cefaclor $(30 \mu \mathrm{g})$, penicillin-G (10units) and ampicillin $(10 \mu \mathrm{g})$ by modified Kirby-Bauer disc diffusion method on Mueller Hinton agar (MHA) containing 5\% defibrinated sheep blood under standard conditions in accordance to latest CLSI guidelines.

This result is in accordance with study conducted by Rijal KR et al ${ }^{[23]}$, Metin Dogan et al ${ }^{[24]}$, Shet et $\mathrm{al}^{[12]}$, Haczynski J et al ${ }^{[25]}$ and Capoor MR et al ${ }^{[26]}$ respectively.

It was also found that 13 isolates $(61.9 \%)$ were sensitive to erythromycin $(15 \mu \mathrm{g})$ out of total 21 isolates and resistant to $8(38.1 \%)$. This result is in accordance with study conducted by Capoor MR et al [26] who showed $29.4 \%$ resistance to erythromycin and similarly $23.7 \%$ by Rondini $G$ et al ${ }^{[27]}$ respectively.

Also it was observed that 15 isolates $(71.4 \%)$ out of total 21 isolates were sensitive to azithromycin $(15 \mu \mathrm{g})$ and were resistant in case of 6 isolates $(28.6 \%)$ which is similar to study conducted by Rijal KR et al ${ }^{[23]}$. Tetracycline $(30 \mu \mathrm{g})$ was sensitive to 7 isolates $(33.3 \%)$ out of total 21 isolates and resistant in case of 14 isolates $(66.7 \%)$ respectively. Some previous studies also showed high rate of tetracycline resistance conducted by Jasir A et al ${ }^{[28]}$ showing $42 \%$ resistance by standard disc diffusion method as well as $100 \%$ resistance shown by study conducted by Camara $\mathrm{M}$ et al ${ }^{[29]}$ and $18 \%$ resistance by Devrim Dunda et al ${ }^{[30]}$ respectively.

\section{Conclusions:}

The findings in our study were consistent with the view that Streptococcus pyogenes infection in children should not be neglected. This study highlights the regular screening and the importance of regular surveillance to keep GABHS in check and to control the development of non-supportive sequelae, by treating children early with appropriate antibiotics by routine culture and sensitivity and also highlights upcoming drug resistance to the commonly used antibiotics which may be due to injudicious and excessive use of antibiotic therapy without following proper antibiotic policy.

\section{References:}

[1] Michael AG, Diagnosis and Treatment of Pharyngitis in Children, Pediatr Clin N Am, 52, 2005, $729-47$.

[2] Abdulwahab MA T, Hani O, A study of group A streptococcal bacterial isolation from children less than 12 years with acute tonsillitis, pharyngitis and healthy primary school children, J Family Community Med, 9(2), 2002, 23-6.

[3] Ozturk CE, Yavuz T, Kanya D, Yucel M, The rate of asymptomatic throat carriage of GAS in school children and associated ASO titre in Duzu Turkey, Jpn J Infect Dis, 57(6), 2004, 27-32.

[4] Azavedo De, Yeung RH, Bast DJ, Duncan CL, Borgia SB, Low DE, Prevalence and mechanism of macrolide resistance in clinical isolates of group A streptococci from Ontario, Canada, Antimicrob Agents Chemother,43, 1999, 2144-7.

[5] Giovanetti E, Montanari MP, Mingoia M, and Varaldo PE, Phenotypes and genotypes of erythromycin-resistant Streptococcus pyogenes strains in Italy and heterogeneity of inducibly resistant strains, Antimicrob Agents Chemother, 43, 1999, 1935-40.

[6] Jasir A, and Schalen C, Survey of macrolide resistance phenotypes in Swedish clinical isolates of Streptococcus pyogenes, J Antimicrob Chemother, 41, 1998, 135-37.

[7] Brahmadathan KN, Gladstone P. Microbiological diagnosis of streptococcal pharyngitis with its lacunae and complications, Indian J Med Microbiol,24(2), 2006, 92-6.

[8] Ross PW, Streptococcus and Enterococcus, in: Collee JG, Fraser AG (Eds.), McCartney Mackie Practical Medical Microbiology, 14 (New Delhi: Elsevier publication, 2011) 263-6.

[9] Mathur NB, Bacteriological examination of pharyngeal secretions, Indian Pediatr,29, 1992, 1071-3.

[10] MacFaddin JF, Biochemical Tests for Identification of Medical Bacteria, (New York: Lippincott Williams and Wilkins, 2000).

[11] Clinical and Laboratory Standards Institute, Perfomance standards for antimicrobial susceptibility testing; Twenty - first Informational Supplement. M100-S21. CLSI, 2011. Villanova Pa.

[12] Shet A, Kaplan E, Addressing the Burden of Group A Streptococcal Disease in India, Indian J Pediatr,71, 2004, 41-6.

[13] Pichichero ME, Group A streptococcal tonsillopharyngitis: cost effective diagnosis and treatment, Ann Emerg Med, 25(3), 1995, 390-403.

[14] Ravi KB, Dudala SR, Rao AR. Kuppuswamy's socioeconomic status scale- A revision of Economic parameter for 2012, Int J of Res and Development of Health, 1(1), 2013, 2-4.

[15] Bassili A, Barakat S, Sawaf GE, Zaher S, Zaki A, Din Saleh EE, Identification of clinical criteria for group A-beta hemolytic streptococcal pharyngitis in children living in a rheumatic fever endemic area, J Trop Pediatr , 48(5),2002, $285-93$.

[16] Giir E, Akkus S, Arvas A, Giizeloz S, Can G, Diren S, Prevalence of positive throat cultures for group A beta-hemolytic streptococci among school children in Istanbul, Indian Pediatr, 39, 2002, 569-73.

[17] Gupta R, Prakash K, Kapoor AK, Subclinical group A streptococcal throat infection in school children, Indian Pediatr , 29(12), 1992, 1491-4.

[18] Lin MH, Fong WK, Chang PF, Yen CW, Hung KL, Lin SJ, Predictive value of clinical features in differentiating group A betahemolytic streptococcal pharyngitis in children, J Microbiol Immunol Infect, 36(1), 2003, 21-5.

[19] Davies HD, Mc Geev Allison, Schwartz Benjamin, Green Karen, Cann Darlene, Simmon Andrew, Invasive group A streptococcal infection in Ontario Canada, N Engl J Med, 1996, 547-54.

[20] Gunnarssu RK, Holm SE, Soderstrom M, The prevalence of beta haemolytic streptococci in throat specimens from healthy childrens and adults and its implications for the clinical value of throat cultures, Scand J Prim Health Care, 15(3), 1997, 149-55.

[21] Moses EA, Goldberg Sara, Korenman Zinaida, Ravins Miriam, Hanski Emmanuel, Shapiro Mervyn, Invasive Group A Streptococcal Infections, Israel, Emerg Infect Dis, 8(4), 2002, 421-426.

[22] Madha M S, Streptococcal throat infection among Yemeni children, Iraqi Journal Sci, 50(1), 2009, 126-35. 
[23] Rijal KR, Dhakal N, Shah RC, Timilsina S, Mahato P, Thapa S, Antibiotic susceptibility of group A Streptococcus isolated from throat swab culture of school children in Pokhara, Nepal, 11(4), 2009, 238-40.

[24] Metin Doğan, Özlem Aydemir, Şükrü Nail Güner, Bahadır Feyzioğlu, Mahmut Baykan, Antibiotic Susceptibility of Group A beta hemolytic Streptococci Isolated From Tonsillar Swab Samples in 5-15 Years Old Children, Eur J Gen Med, 11(1), 2014, 29-32.

[25] Haczyński J, Bardadin J, Gryczyńska D, Gryczyński M, Gołabek W, Kawalski H, A comparative study of cefaclor versus amoxicillin/clavulanate in tonsillopharyngitis, Med Sci Monit, 7(5), 2001, 1016-22.

[26] Capoor MR, Nair D, Deb M, Batra K, Aggarwal P, Resistance to erythromycin and rising penicillin MIC in Streptococcus pyogenes in India, Jpn J Infect Dis, 59(5), 2006, 334-6.

[27] Rondini G, Cocuzza CE, Cianflone M, Lanzafame A, Santini L, Mattina R, Bacteriology and clinical efficacy of various antibiotics used in the treatment of streptococcal pharyngitis in Italy. An epidemiological study, Int J Antimicrob Agents, 18(1), 2001, 9-17.

[28] Jasir A, A Tanna, A Noorani, A Mirsalehian, A Efstratiou, C Schaleni, High Rate of Tetracycline Resistance in Streptococcus pyogenes in Iran: an Epidemiological Study, J Clin Microbiol, 38(6), 2000, 2103-2107.

[29] Camara M, Assane Dieng and Cheikh Saad Bouh Boye, Antibiotic Susceptibility of Streptococcus Pyogenes isolated from Respiratory Tract Infections in Dakar, Senegal, Microbiology Insights, 6, 2013, 71-75.

[30] Devrim Dundar, Murat Sayan, Gulden Sonmez Tamer, Macrolide and Tetracycline Resistance and M Type Distribution of Streptococcus pyogenes isolates recovered from Turkish Patients, Microb Drug Resist, 16(4), 2010, 279-284. 